\title{
A 2-Week Course of Naproxen for Heterotopic Ossification Prophylaxis Is Effective Following Hip Arthroscopy for Femoroacetabular Impingement
}

\author{
Alexander J. Mortensen, B.S., Dillon C. O’Neill, M.D., Temitope F. Adeyemi, M.P.H., \\ Travis G. Maak, M.D., and Stephen K. Aoki, M.D.
}

\begin{abstract}
Purpose: To evaluate the effectiveness of 2 weeks of naproxen prophylaxis for heterotopic ossification (HO) after primary hip arthroscopy for femoroacetabular impingement (FAI). Methods: All consecutive hip arthroscopy cases by a single surgeon from January 1, 2015, to December 31, 2016, were retrospectively reviewed. Cases were included if they met the following criteria: (1) Primary hip arthroscopy, including femoral neck osteoplasty, for FAI; (2) naproxen prophylaxis for 2 weeks' duration; and (3) radiographic follow-up at a minimum of 3 months postoperatively. Radiographic imaging for each patient was evaluated for the presence of $\mathrm{HO}$, and independent radiology reports were used to confirm patients with HO. Maximal size of $\mathrm{HO}$ was measured and classified according to the Brooker criteria. Demographic and operative variables of the patients included in this study who received naproxen prophylaxis for 2 weeks were formally compared with a cohort of patients who received naproxen prophylaxis for 3 weeks in a previous randomized controlled trial. Results: A total of 185 patients who received naproxen prophylaxis for 2 weeks were included in the study, 5 of whom developed HO (3\%). A total of 48 patients who received naproxen prophylaxis for 3 weeks were included for comparison, 2 of whom developed HO (4\%). There was no significant difference in the rate of HO formation between 2 weeks and 3 weeks of naproxen prophylaxis $(P=.597)$. All 7 cases of HO were classified as Brooker class 1. Conclusions: The observed rate of postoperative heterotopic ossification after 2 weeks of naproxen prophylaxis in this study was equivalent to that observed after 3 weeks' prophylaxis in a previously published study. Two weeks of naproxen prophylaxis may be a sufficient treatment course for the prevention of heterotopic ossification after primary hip arthroscopy for FAI. Level of Evidence: Level III - Retrospective Comparative Study.
\end{abstract}

$\mathbf{H}$ eterotopic ossification ( $\mathrm{HO})$ is a known complication of hip arthroscopy that results from dysregulated osteogenic signaling within damaged soft tissues surrounding hip musculature. ${ }^{1}$ There are numerous

From the School of Medicine (A.J.M.) and Department of Orthopaedic Surgery (D.C.O., T.F.A., T.G.M., S.K.A.), University of Utah, Salt Lake City, Utah, U.S.A.

The authors report the following potential conflicts of interest or sources of funding: Disclosures: T.M. is a paid speaker for Arthrex Medical, outside of the submitted work. S.A. is a paid education consultant for Stryker Medical, outside of the submitted work. Full ICMJE author disclosure forms are available for this article online, as supplementary material.

This study was performed at the University of Utah in Salt Lake City, Utah. Received December 9, 2019; accepted July 10, 2020.

Address correspondence to Stephen K. Aoki, M.D., Department of Orthopaedic Surgery, 590 Wakara Way, Salt Lake City, UT 84108. E-mail: stephen.aoki@hsc.utah.edu

(C) 2020 THE AUTHORS. Published by Elsevier Inc. on behalf of the Arthroscopy Association of North America. This is an open access article under the CC BY-NC-ND license (http://creativecommons.org/licenses/by-nc-nd/4.0/).

2666-061X/191511

https://doi.org/10.1016/j.asmr.2020.07.016 signaling pathways and multiple cell types involved in the pathogenesis of $\mathrm{HO}^{2}$ Previous research has shown $\mathrm{HO}$ is radiographically visible as early as 1 to 2 months following soft-tissue injury. ${ }^{3,4}$ Surveillance for $\mathrm{HO}$ should continue for 9 weeks following hip arthroscopy, although HO will continue to mature and may become symptomatic past this time point. ${ }^{5}$ Without intervention, $\mathrm{HO}$ is reported to occur at a rate between $6 \%$ and $44 \%$ of patients who undergo hip arthroscopy. ${ }^{5-9}$ In the last 10 years, nonsteroidal anti-inflammatory drug (NSAID) prophylaxis, which disrupts inflammatory prostaglandin signaling resulting from operative damage, ${ }^{3}$ has been shown to decrease the incidence of $\mathrm{HO}$ in several retrospective studies. ${ }^{8-11}$ These findings were corroborated by a double-blind, randomized, placebo-controlled trial, which demonstrated that 3 weeks of naproxen postoperatively reduced the rate of $\mathrm{HO}$ in hip arthroscopy versus placebo from $46 \%$ to $4 \% .^{12}$

Although NSAIDs are well-tolerated by most individuals, adverse effects can occur even with short-term use. Documented side effects associated with HO 
prophylaxis about the hip include dyspepsia, nausea, gastrointestinal bleeding, and acute renal failure. ${ }^{9,13}$ Medication side effects can be reduced by limiting the dose, frequency, or duration of medication administration. The ideal regimen of NSAID prophylaxis would consist of the least potent NSAID and shortest duration of therapy while maintaining maximal effectiveness in preventing ectopic bone formation. Importantly, with the exception of a single study by Rath et al. ${ }^{14}$ using etodolac, previous studies have used durations of NSAID prophylaxis of at least 3 weeks. It is uncertain whether a shorter duration of naproxen prophylaxis is sufficient to achieve equivalent results.

The purpose of this study was to evaluate the effectiveness of 2 weeks of naproxen prophylaxis for HO after primary hip arthroscopy for femoroacetabular impingement (FAI). Our hypothesis was that naproxen prophylaxis of 2 weeks duration would be equally as effective in preventing HO when compared with 3 weeks of naproxen prophylaxis as demonstrated previously.

\section{Methods}

Each author certifies that his or her institution approved the human protocol for this investigation that all investigations were conducted in conformity with ethical principles of research, and that informed consent for participation in the study was obtained. This study was performed under the University of Utah Institutional Review Board-approved protocol \#71733.

All hip arthroscopy cases performed by the senior author (S.K.A.) between January 1, 2015, and December 31, 2016, were retrospectively reviewed in the electronic medical record. During this time period, all patients of the senior author who did not have a contraindication to NSAID prophylaxis were prescribed 2 weeks of an NSAID, most commonly naproxen. Naproxen prophylaxis consisted of naproxen $500 \mathrm{mg}$ twice daily for 2 weeks beginning the day after surgery. Contraindications to NSAID prophylaxis during the study time period were history of gastrointestinal disorders and/or upset with NSAID use, a documented allergy to any NSAID, and a history of cardiovascular disease.

Of the identified cases, patients were included in the study only if they met the following inclusion criteria: (1) primary hip arthroscopy procedure, including femoral neck osteoplasty, with FAI as the main indication; (2) documented 2 weeks of naproxen prophylaxis; and (3) minimum of 3 months radiographic follow-up postoperatively. Individual hips were treated as separate cases. Patients were included in the study if they were treated for other pathology in addition to FAI during the procedure. Cases were excluded if patients received an NSAID other than naproxen. Cases with isolated indications other than FAI and revision cases were excluded. For included patients, pre- and perioperative demographic data were collected from the electronic medical record, including age, sex, and body mass index.

Patients were diagnosed with FAI based on a history of hip pain, exacerbation of hip pain with the anterior impingement maneuver on physical examination, and radiographic findings including decreased femoral head-neck offset consistent with cam deformity, increased center edge angle $>40^{\circ}$ consistent with pincer deformity, and evidence of labral tear on magnetic resonance imaging. Indication for surgery included patients with a diagnosis of FAI who did not respond to nonoperative treatment. Surgical technique included application of traction to the operative lower extremity to achieve adequate distraction to safely instrument the hip joint, an interportal capsulotomy through connection of an anterolateral and modified anterior portal, labral repair with suture anchors, acetabuloplasty, femoroplasty, and routine capsular repair.

Preoperative radiographic assessment included anteroposterior, frog-leg lateral, and modified false-profile radiographic views of the operative hip. These preoperative radiographs were reviewed for each patient to provide effective comparison with postoperative hip radiographs. Postoperative evaluation consisted of independent review of postoperative anteroposterior, frog-leg lateral, and false-profile radiographs. All postoperative radiographs were independently reviewed by 2 of the study authors (S.K.A., A.J.M.) for the presence of HO. Cases of HO were evaluated separately on each radiographic view to ensure diagnostic accuracy and were then confirmed by a retrospective review of official radiology reports available in the electronic medical record.

All cases of $\mathrm{HO}$ were classified according to the Brooker criteria and measured on the radiographic view, which provided the largest 2 -dimensional area. ${ }^{15}$ Class I describes bone islands within the surrounding soft tissues of the hip, class II describes bone growth from the pelvis or proximal end of the femur with $\geq 1 \mathrm{~cm}$ between opposing bone ends, class III describes bone growth from the pelvis or proximal end of the femur with $<1 \mathrm{~cm}$ between opposing bone ends, and class IV describes complete ankyloses of the hip joint. In addition to classification with Brooker criteria, HO area and length measurements were performed. Area measurements were made by digitally outlining the ectopic bone to provide a calculated area. Maximum 2-dimensional length was measured along the longest axis. Area and length measurements were both performed on the radiographic view that provided the largest value.

All cases with 2 weeks of naproxen prophylaxis who met inclusion criteria were compared with a cohort of patients who underwent hip arthroscopy performed by the same surgeon between August 2011 and April 2013. These cases were part of a previously published double-blind randomized placebo-controlled trial, ${ }^{12}$ 
Fig 1. Flowchart diagram of all hip arthroscopy cases by the senior author who were included and excluded during the study periods of August 2011 to April 2013 and January 2015 to December 2016. (FAI, femoroacetabular impingement; $\mathrm{HO}$, heterotopic ossification; NSAID, nonsteroidal anti-inflammatory drug.)

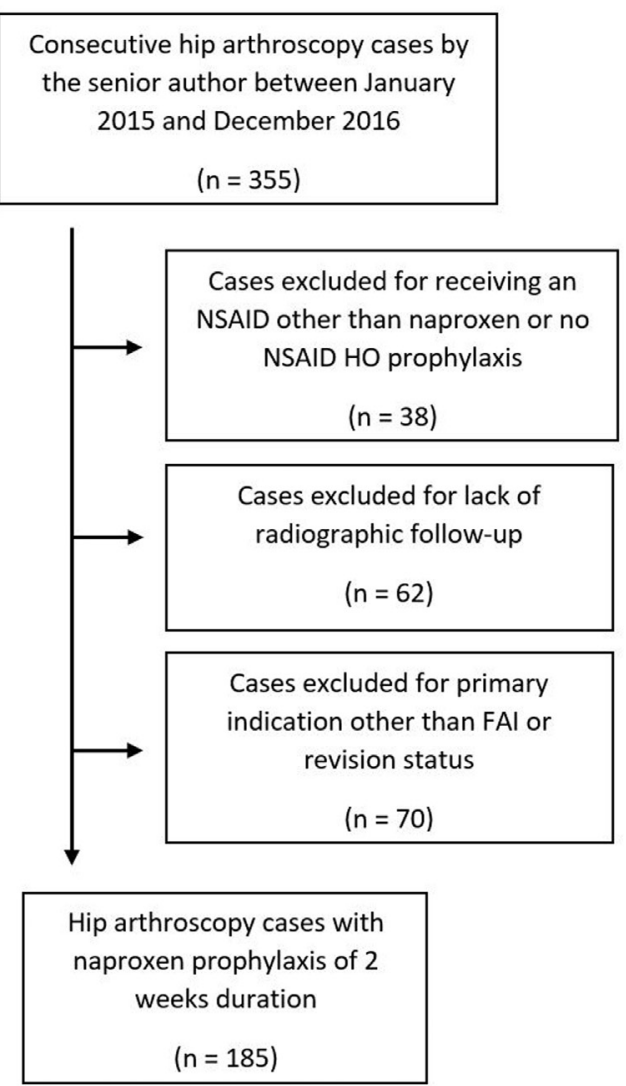

and were all randomized to receive naproxen prophylaxis for 3 weeks' duration. An identical daily dosage and frequency of naproxen was given to each cohort of patients.
Statistical analyses were performed in Excel $\mathrm{X}$ (Microsoft, Redmond, WA) and SPSS 25 (IBM Corp., Armonk, NY). Descriptive statistics were calculated. Kolmogorov-Smirnov tests were used to assess for

Table 1. Demographic and Intraoperative Comparison by Heterotopic Ossification Formation

\begin{tabular}{|c|c|c|c|}
\hline & No Heterotopic Ossification & Heterotopic Ossification & $P$ Value \\
\hline \multicolumn{4}{|l|}{ Demographics } \\
\hline $\mathrm{N}$ (total patients) & $180(97.3 \%)$ & $5(2.7 \%)$ & - \\
\hline Female & $118(65.6 \%)$ & $3(60 \%)$ & 1.000 \\
\hline $\operatorname{Age}^{\dagger \ddagger}$ & $32.7 \pm 11.7$ & $27.5 \pm 10.5$ & .264 \\
\hline Side ${ }^{*}$ & & & .655 \\
\hline Right & $102(56.7 \%)$ & $2(40 \%)$ & \\
\hline Isolated cam resection ${ }^{*}$ & $115(63.9 \%)$ & $3(60 \%)$ & 1.000 \\
\hline Cam and pincer resection* & $65(36.1 \%)$ & $2(40 \%)$ & 1.000 \\
\hline Labral repair & $79(43.9 \%)$ & $1(20 \%)$ & .391 \\
\hline No. labral repair anchors ${ }^{\dagger \dagger}$ & $2.4 \pm 0.8$ & 2 & .561 \\
\hline Capsular closure & $100 \%$ & $100 \%$ & - \\
\hline No. capsular closure sutures ${ }^{\dagger \ddagger}$ & $5.4 \pm 1.2$ & $5.2 \pm 1.1$ & .864 \\
\hline
\end{tabular}


Table 2. Cases of Heterotopic Ossification

\begin{tabular}{|c|c|c|c|c|c|}
\hline & Case \# 1 & Case \#2 & Case \#3 & Case \#4 & Case \#5 \\
\hline$\overline{\text { Sex }}$ & Female & Male & Male & Female & Female \\
\hline BMI & 32.7 & 26.7 & 19.2 & 22.5 & 29.1 \\
\hline Age & 41 & 19 & 21 & 19 & 35 \\
\hline Heterotopic ossification area, $\mathrm{mm}^{2}$ & 55.6 & 17.1 & 8.5 & 31.3 & 14.7 \\
\hline Maximum 2-dimensional size, $\mathrm{mm}$ & 11 & 8 & 7 & 10 & 5 \\
\hline Brooker class & I & I & I & I & I \\
\hline
\end{tabular}

NOTE. Each case of heterotopic ossification formed in patients who received naproxen prophylaxis for 2 weeks' duration. BMI, body mass index.

normality for each continuous variable. Continuous variables were compared using either a Mann-Whitney $U$ test or independent samples $t$ test depending on the normality of the sample. Categorical variables were compared using Pearson $\chi^{2}$ and Fisher exact tests.

\section{Results}

A total of 355 consecutive cases between January 1 , 2015, and December 31, 2016, were identified and evaluated against the inclusion and exclusion criteria of the study. Cases were excluded due to a primary indication other than FAI or revision status (19\%), NSAID prophylaxis other than naproxen or no NSAID prophylaxis due to a contraindication $(11 \%)$, or lack of radiographic follow-up of equal to or longer than 3 months postoperatively (17\%). A total of 185 patients received naproxen prophylaxis of 2 weeks duration and were included in the study (Fig l).

Mean radiographic follow-up for the cohort was $13.4 \pm 9.4$ months. HO was identified in $5(2.7 \%)$ patients, whereas 180 patients $(97.3 \%)$ did not develop HO. Demographic and surgical technique information for both groups is presented in Table 1 . There were no statistically significant differences between groups. The authors agreed on the presence or absence of $\mathrm{HO}$ in each case, and there was no disagreement between author evaluation and radiology reports.

Patient characteristics for individuals who received 2 weeks of naproxen prophylaxis and formed $\mathrm{HO}$ is presented in Table 2. Mean maximal area of HO was $25.4 \mathrm{~mm}^{2}$, and Figure 2 demonstrates the largest case of HO $\left(55.6 \mathrm{~mm}^{2}\right)$ in this group. All 5 cases of HO in the 2-week naproxen prophylaxis cohort were classified as Brooker grade 1.

A total of 48 hip arthroscopy cases between August 2011 and April 2013 received naproxen prophylaxis for 3 weeks' duration (Fig 1). ${ }^{12}$ Demographic and operative data for these cases were available for comparison. Results of comparison between patients in the 2 and 3 weeks of naproxen prophylaxis groups are presented in Table 3. The rate of HO formation was $4.2 \%$ in patients who received naproxen prophylaxis for 3 weeks and was not significantly different from the rate observed in patients receiving naproxen prophylaxis of 2 weeks $(P=.635)$. Mean 2 -dimensional HO size was $8.2 \mathrm{~mm}$ in patients with 2 weeks' naproxen prophylaxis and $11.6 \mathrm{~mm}$ in patients with 3 weeks' naproxen prophylaxis. Compared with the 3 -week naproxen prophylaxis group, the 2 -week prophylaxis group had a greater percentage of patients who underwent isolated cam resection $(64 \%$ vs $27 \% ; P<.001)$ and a lower percentage of patients who underwent labral repair (43\% vs $65 \% ; P=.008)$.

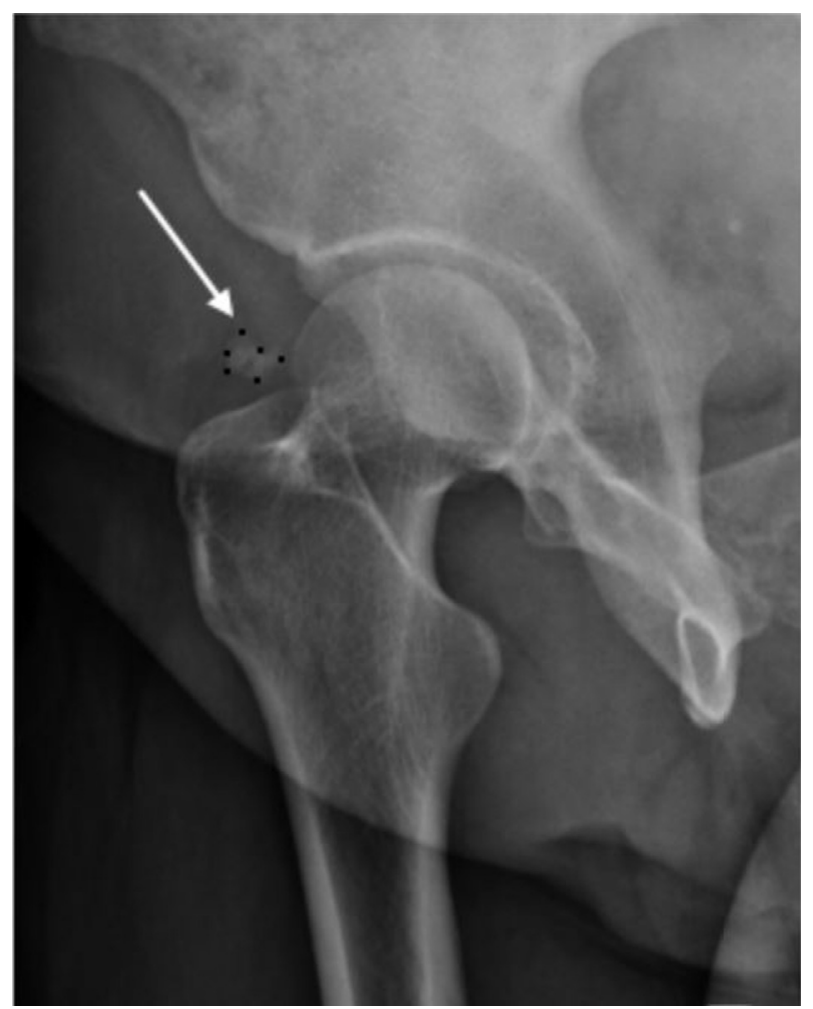

Fig 2. Modified false-profile radiograph of the right hip taken 6 months postoperatively. Arrow points to heterotopic ossification outlined in black dots located anterior and superior to the greater trochanter $\left(\right.$ area $\left.=55.6 \mathrm{~mm}^{2}\right)$. Heterotopic ossification area was measured by digitally outlining the ectopic bone to provide a calculated area, and the maximum 2-dimensional length was measured along the longest axis. Area and length were measured on the radiographic view that provided the largest value. 
Table 3. Two Weeks' Versus Three Weeks' Duration of Naproxen HO Prophylaxis

\begin{tabular}{|c|c|c|c|}
\hline & 2 Weeks' Naproxen Prophylaxis & 3 Weeks' Naproxen Prophylaxis & $P$ Value \\
\hline \multicolumn{4}{|l|}{ Demographic } \\
\hline $\mathrm{N}$ (total patients) & 185 & 48 & - \\
\hline Female, \%* & $65 \%$ & $58 \%$ & .401 \\
\hline \multicolumn{4}{|l|}{ Intraoperative } \\
\hline Cam and/or pincer resection ${ }^{\S}$ & $100 \%$ & $96 \%$ & .042 \\
\hline Isolated cam resection ${ }^{*}$ & $64 \%$ & $27 \%$ & $<.001$ \\
\hline Capsular closure ${ }^{\S}$ & $100 \%$ & $98 \%$ & .206 \\
\hline \multicolumn{4}{|l|}{ Radiographic assessment } \\
\hline Cases of $\mathrm{HO}$ & 5 & 2 & - \\
\hline$\% \mathrm{HO}^{\S}$ & $2.7 \%$ & $4.2 \%$ & .635 \\
\hline Mean 2-dimensional size & 8.2 & 11.6 & - \\
\hline
\end{tabular}

NOTE. Demographics, intraoperative variables, and radiographic assessment comparing 2 vs. 3 weeks of naproxen heterotopic ossification prophylaxis following hip arthroscopy.

HO, heterotopic ossification.

*Pearson $\chi^{2}$ test.

${ }^{\dagger}$ Mean \pm standard deviation.

${ }^{\ddagger}$ Mann-Whitney U test.

${ }^{\S}$ Fisher exact test.

\section{Discussion}

This study demonstrated that the rate of $\mathrm{HO}$ with 2 weeks of naproxen $(2.7 \%)$ is equivalent to the rate of $\mathrm{HO}$ with 3 weeks of naproxen $(4.2 \%)$. An identical daily dosage and frequency of naproxen was given to patients in both cohorts, and hip arthroscopy procedures were performed by the same surgeon in all cases. Direct comparison between groups showed only slight differences. There were no significant differences in sex, age, or rate of capsular closure. Although there were significant differences in rate of isolated cam versus mixed-type FAI resection and labral repair, the surgical technique of the senior author was otherwise very similar during both study cohort time periods. Ultimately, these cohorts are a reasonable comparison, suggesting the efficacy of naproxen prophylaxis for a reduced duration of 2 weeks.

Previous retrospective studies have shown similar rates of HO following hip arthroscopy with varying durations of NSAID prophylaxis. Randelli et al. previously reported no HO formation with 3 weeks of NSAID prophylaxis in 285 patients who underwent hip arthroscopy for FAI. ${ }^{8}$ Indomethacin for 4 days, followed by naproxen for 30 days, has formerly been shown to reduce the rate of $\mathrm{HO}$ formation to $1.8 \%$ in primary hip arthroscopy. ${ }^{10}$ Beckmann et al. showed the rate of HO to decrease from $25.0 \%$ in cases with no NSAID prophylaxis versus $5.6 \%$ of cases with NSAID prophylaxis. ${ }^{9}$ In addition, Rath et al. demonstrated no HO formation in 63 patients receiving etodolac prophylaxis for 2 weeks beginning the day after surgery. ${ }^{14}$

All 7 cases of HO from both the 2- and 3-week naproxen prophylaxis cohorts were classified as Brooker grade I. Due to a limited number of $\mathrm{HO}$ cases, there was no formal statistical comparison made between the sizes of $\mathrm{HO}$ in both groups. However, mean 2-dimensional size were very comparable between groups, being $8.2 \mathrm{~mm}$ with 2 weeks of naproxen and $11.6 \mathrm{~mm}$ with 3 weeks of naproxen. Although there are several studies evaluating NSAID prophylaxis on HO incidence rates, there is limited literature directly evaluating the effect NSAIDs have on the size of HO. Beckmann et al. observed a smaller average 2-dimensional $\mathrm{HO}$ size in the naproxen group when compared with the placebo group. In addition, in the study of Beckmann et al., all cases of $\mathrm{HO}$ formed in the naproxen group were Brooker grade I, whereas 7 cases of $\mathrm{HO}$ in the placebo group were Brooker grade II. ${ }^{12}$ Furthermore, findings from Bedi et al. showed a greater percentage $(83 \%)$ of Brooker grade I in the indomethacin plus naproxen group, possibly indicating the role of additional NSAID prophylaxis in reducing the severity and size of $\mathrm{HO}$ formation. ${ }^{10}$

Furthermore, the senior author of this paper performed all hip arthroscopy surgeries in both of the studied cohorts. Two additional factors were emphasized in practice throughout the period of this study that may contribute to the further decrease in $\mathrm{HO}$ incidence relative to the cohort who received 3 weeks of naproxen. First, patient education was improved and counseling was performed routinely with all patients at the time of surgery to stress the importance of NSAIDs in preventing $\mathrm{HO}$ formation. Oftentimes patients may be unaware of the prophylactic role NSAIDs play following hip arthroscopy and may be more focused on the role of NSAIDs in reducing symptomatic pain and inflammation. Proper patient education on the prophylactic role of NSAIDs can facilitate greater compliance to a scheduled 
medication regimen. Second, careful consideration of the technical aspects of evacuating bone debris following hip osteochondroplasty should be considered to minimize HO. Remaining bone debris may serve as a nidus for the development of HO. Therefore, careful evacuation of all accessible bone debris following osteochondroplasty may be an important technical aspect performed by surgeons in the intraoperative period to reduce ectopic bone formation.

\section{Limitations}

Our study has several limitations. Our groups are unequal; the 2 -week group included 185 patients whereas the 3-week group included 48 patients. First, radiographic follow-up was incomplete in $17 \%$ of the patients during this study period (62 of 355); the sample size with available radiographic follow-up during the study period provides a large sample to effectively power this study. Second, radiographic follow-up was defined as a time interval of greater than 3 months postoperatively. While it is possible for $\mathrm{HO}$ to mature and become apparent radiographically past this time period, both 3 months and 9 weeks have previously been used as follow-up interval cutoffs to effectively evaluate for $\mathrm{HO},{ }^{5,12}$ In addition, the cohort of patients who received 3 weeks of naproxen prophylaxis were previously evaluated based on a minimum radiographic follow-up of 75 days. We chose a minimum follow-up of 3 months because it is a reasonable comparison and slightly longer time period. Third, the 2 study cohorts included patients from different time periods. The total time period spanning both studies is 4 years and 5 months, and the 2 cohorts are separated by a year and 8 months, which has the potential of introducing confounding variables. Increased surgeon experience throughout this time may have led to improved technique, including improved attention to careful evacuation of accessible bone debris following osteochondroplasty, as previously discussed. A comparison of intraoperative variables between the 2 cohorts showed there were significant differences in rates of isolated cam resection and labral repair. However, ultimately all patients in the study underwent hip arthroscopy indicated for treatment of FAI by the same surgeon, and there were no major changes in the surgical technique of the senior author during this time period. Furthermore, demographic variables of each cohort showed no significant differences. Finally, assessment was limited to plain radiographs as the only imaging modality used in this study, as plain radiography has good sensitivity in detecting $\mathrm{HO}$ and is routinely performed in postoperative evaluation of FAI.

\section{Conclusions}

The observed rate of postoperative HO after 2 weeks of naproxen prophylaxis in this study was equivalent to that observed after 3 weeks of prophylaxis in a previously published study. Two weeks of naproxen prophylaxis may be a sufficient treatment course for the prevention of HO after primary hip arthroscopy for FAI.

\section{References}

1. Balboni TA, Gobezie R, Mamon HJ. Heterotopic ossification: Pathophysiology, clinical features, and the role of radiotherapy for prophylaxis. Int J Radiat Oncol 2006;65: 1289-1299.

2. Ranganathan K, Loder S, Agarwal S, et al. Heterotopic ossification: Basic-science principles and clinical correlates. J Bone Joint Surg Am 2014;97:1101-1111.

3. Kaplan FS, Glaser DL, Hebela N, Shore EM. Heterotopic ossification. J Am Acad Orthop Surg 2004;12:116-125.

4. Orzel JA, Rudd TG. Heterotopic bone formation: Clinical, laboratory, and imaging correlation. J Nucl Med 1985;26: $125-132$.

5. Rath E, Sherman H, Sampson TG, Ben Tov T, Maman E, Amar E. The incidence of heterotopic ossification in hip arthroscopy. Arthrosc J Arthrosc Relat Surg 2013;29:427-433.

6. Clohisy JC, Zebala LP, Nepple JJ, Pashos G. Combined hip arthroscopy and limited open osteochondroplasty for anterior femoroacetabular impingement. J Bone Joint Surg Am 2010;92:1697-1706.

7. Larson CM, Giveans MR. Arthroscopic management of femoroacetabular impingement: Early outcomes measures. Arthroscopy 2008;24:540-546.

8. Randelli F, Pierannunzii L, Banci L, Ragone V, Aliprandi A, Buly R. Heterotopic ossifications after arthroscopic management of femoroacetabular impingement: The role of NSAID prophylaxis. J Orthop Traumatol 2010;11:245-250.

9. Beckmann JT, Wylie JD, Kapron AL, Hanson JA, Maak TG, Aoki SK. The effect of NSAID prophylaxis and operative variables on heterotopic ossification after hip arthroscopy. Am J Sports Med 2014;42:1359-1364.

10. Bedi A, Zbeda RM, Bueno VF, Downie B, Dolan M, Kelly BT. The incidence of heterotopic ossification after hip arthroscopy. Am J Sports Med 2012;40:854-863.

11. Yeung M, Jamshidi S, Horner N, Simunovic N, Karlsson J, Ayeni OR. Efficacy of nonsteroidal anti-inflammatory drug prophylaxis for heterotrophic ossification in hip arthroscopy: A systematic review. Arthroscopy 2016;32:519-525.

12. Beckmann JT, Wylie JD, Potter MQ, Maak TG, Greene TH, Aoki SK. Effect of naproxen prophylaxis on heterotopic ossification following hip arthroscopy. J Bone Joint Surg Am 2015;97:2032-2037.

13. Fransen M, Anderson C, Douglas J, et al. Safety and efficacy of routine postoperative ibuprofen for pain and disability related to ectopic bone formation after hip replacement surgery (HIPAID): Randomised controlled trial. BMJ 2006;333:519.

14. Rath E, Warschawski Y, Maman E, et al. Selective COX-2 inhibitors significantly reduce the occurrence of heterotopic ossification after hip arthroscopic surgery. Am J Sports Med 2016;44:677-681.

15. Brooker AF, Bowerman JW, Robinson RA, Riley LH. Ectopic ossification following total hip replacement. Incidence and a method of classification. J Bone Joint Surg Am 1973;55:1629-1632. 